\title{
Seamless Mobility Management between IP-based Networks
}

\author{
M. M. A. Khan, Omar Arafat", K. Dimyati, Fatima Seeme \\ Department of Electrical Engineering, University of Malaya, Kuala Lumpur, Malaysia \\ University of Malaya, Kuala Lumpur, 50603, Malaysia
}

\begin{abstract}
This article represents an approach of providing seamless services to the mobile consumer in an area having convergence of different Radio Access Technologies (RATs). In particular, the proposed framework is capable of managing session mobility between General Packet Radio Service (GPRS) and Worldwide Interoperability for Microwave Access (WiMAX) at the core network level. Within this interworking architecture, IP Multimedia Subsystem (IMS) was applied as a common platform at the application layer for session mobility and Mobile IP was applied at the network layer to maintain IP mobility. The unique contribution of this architecture is the hypothetical user mobile device having multiple transceivers along with intelligence of making decisions of handover. The article comes to an end by narrating the potential advantages of the proposed approach in eliminating the packet loss due to handover.
\end{abstract}

Keywords IMS, Intersystem Handover, Mobile IP, OPNET Modeler, WiMAX

\section{Introduction}

The upcoming Internet Protocol (IP) based $4^{\text {th }}$ Generation (4G) networks should have the capability of providing ubiquitous access to internet for the roaming users. The gradually increasing demand for internet and data access is the key motivation for the operators to search for better RATs to fulfil that demand and provide services to the consumers with more efficiently at higher data rate. The well established 2.5G network GPRS made it possible to provide packet data services over the traditional Circuit Switched (CS) system Global System for Mobile telecommunications (GSM). However, GPRS can only support a very low data rate which is not sufficient for data-intensive applications. Later on, the $3^{\text {rd }}$ Generation (3G) networks like Universal Mobile Telecommunications System (UMTS), Code Division Multiple Access (CDMA) and others were launched with the capability of providing higher data rate than that of GPRS. But still it was not sufficient to satisfy the consumer demand. Afterwards, the wireless technologies were introduced with a hope of providing data rates higher than ever possible.

The WiMAX, IEEE 802.16-2004 was standardized to support high data rates up to $63 \mathrm{Mbps}$ for Down Link (DL) and $28 \mathrm{Mbps}$ for Up Link (UL), Quality of Service (QoS), scalability and security schemes[1]. To support mobility to

* Corresponding author:

omararafat@siswa.um.edu.my (Abdullah Omar Arafat)

Published online at http://journal.sapub.org/ijnc

Copyright (C) 2012 Scientific \& Academic Publishing. All Rights Reserved the end users at vehicular speed, this standard was upgraded to IEEE 802.16e[2]. It has mobility supporting handover schemes with latencies less than $50 \mathrm{~ms}[3]$. However, the standard does not fully define the complete network infra-structure which is a critical stumbling block for deploying WiMAX of its own and still remains as a far future. Nevertheless, WiMAX can be interworked with the available cellular networks.

This convergence of IP-base cellular and wireless networks brings in new challenges in terms of quality and capacity. Henceforth, the operators are looking for openings of intersystem service handover to utilize the spectrum more efficiently. This article represents an interworking architecture between WiMAX and GPRS capable of maintaining continuity of an on-going session keeping the IP address of the consumer as static.

The reminder of the article is organised as follows. The next section briefly describes the background and motivation for the proposed architecture. This is followed by a short introduction of the architecture. The interworking architectture and its working principle are narrated briefly. The fourth section describes about the simulation environment built by OPNET Modeler 14.5 and the results of simulation. Finally we discuss about the results of simulation and come to the end for this article with short concluding remarks and acknowledgement of the research work.

\section{Background and Motivation}

The available access technologies differ in terms of spectrums, bandwidths, media access technologies, security 
mechanisms and so on. Hence, service handover from one access technology to another has become a critical issue to be resolved for the upcoming $4 \mathrm{G}$ networks. Until now the resolution of intersystem handover limits to break-beforemake type of handover and providing of seamless service still requires further steps for finding the proper way out. For example, the handover between GPRS and UMTS using compress mode is not seamless and the on-going session is forced to drop. It can also be compared as inter-technology roaming. However, for handover between UMTS and Wireless Local Area Network (WLAN) the data link layer technology is to be changed[4].

The most important issues of intersystem handover are a) session continuity which is of an application layer issue and b) IP management which is of a network layer issue. Some strategies for such handover have been proposed which mainly focused either on the application layer through IMS or on the network layer through Mobile IP (MIP)[5-11]. However, the works presented in[12,13] adopted IMS and MIP together which resulted in better outputs in terms of latency and throughput. The method presented in this paper adopts the similar idea as in[12,13]. However, it has some additional features.

\section{Proposed Method}

The proposed architecture has three main features. Firstly, the architecture applies MIP at the network layer to increase the possibility of mobility between different IP subnets without changing the home IP address. But choosing the version of IP required in-depth investigation. 3GPP IMS was mainly standardised for IP version 6 (IPv6). However, according to the early releases, IMS is also possible to implement using the IP version 4 (IPv4)[17]. The WiMAX can support both IPv4 and IPv6. But the GPRS SGSN does not support the IPv6. Hence, if IPv6 was used there would be an additional latency regarding to Network Address Translation (NAT) every time signal passing through the SGSN. On the other hand, if IPv4 was used for IMS, WiMAX and GPRS, it would become a flat platform and the latency related to NAT would be eliminated. Hence, IPv4 was used in our proposed method. However, MIP assigns two addresses for each user while away from the home network. The Foreign Agent (FA) located at the visiting network assigns a Care-of-Address $(\mathrm{CoA})$ to the user away from home network. The user needs to register the CoA with the Home Agent (HA) located at the home network. The data packets received at the HA from higher-layers are sent to the appropriate FA keeping the higher-layer always in dark about the current location of the user[14]. The second feature of this approach is the use of IMS defined by 3GPP[15] to support session management and negotiation at the application layer. IMS is capable of supporting any type of access technology (e.g. WiMAX, UMTS, GPRS, WLAN and fixed lines) and allows a maximum convergence of services by using the same path of signalling for all applications regardless of the access tech- nologies. The base protocol for IMS is the Session Initiation Protocol (SIP) which is capable of supporting all four kinds of mobility defined by Internet Engineering Task Force (IETF)[16]. Thirdly, a hypothetical mobile device was proposed having multiple transceivers to maintain radio links simultaneously with GPRS and WiMAX. The device would compare strengths of signals received from both networks, keep monitoring and make decisions of handover when appropriate. Hence, the handover would be mobile initiated. The device would break the connection with current network after making a new connection with the targeted network through the other interface resulting in a make-before-break type of handover in the non-overlapping area. While in overlapping area the device would complete the link layer registrations simultaneously with each access technology available there and start getting services by doing service registration through an appropriate interface according to the priority of selection. This approach would eliminate the latency due to link layer registration after handover decision in the overlapping area and the user would need to make the service registration with the new network to get services.

\subsection{Network Architecture}

The proposed overall network, as shown in Fig. 1, comprises of a home network and two visited networks, as the conception of IMS. Each visited network has a Proxy Call Session Control Function (P-CSCF) which is a SIP proxy and can be considered as the first entrance to the IMS terminal. The user device discovers its P-CSCF with either Dynamic Host Configuration Protocol (DHCP) or it is assigned in the Packet Data Protocol (PDP) Context during registration. The P-CSCF appears on the path of all signalling messages compressing and decompressing to authenticate the user and to establish an IP security (IPSec) association when necessary. The Serving Call Session Control Function (S-CSCF), Interrogating- Call Session Control Function (I-CSCF), Home Subscriber Server (HSS) and other IMS components are situated in the home network. S-CSCF is the central node of the signalling plane that performs session control, appears on the path of all signalling messages and is the main entrance to SIP services that uses Diameter Cx and Dx interfaces to get information about user profile from the HSS. HSS is the master database that stores all necessary information about the user and performs authentication and authorization for the user. I-CSCF is another SIP server and its IP address is known to the Domain Name System (DNS) of the domain. This is due to make the I-CSCF available for remote servers to use it as a forwarding point for SIP packets to this domain. The I-CSCF uses the Diameter $\mathrm{Cx}$ interface to retrieve the user location, and then routes the SIP request to its assigned S-CSCF.

For MIP functionality, each visited network would have a FA and the FA for GPRS networks is the Gateway GPRS Support Node (GGSN) and for WiMAX Network, it is the ASN Gateway. FA stores information about visiting users and advertises CoA periodically. The HA situated in the 
home network stores information about users' permanent home address. The permanent home address is used as the destination address for the user trying to communicate with a node and the IP routers forwards all the packets to HA even if the user's current location is away from the home network visiting a different network. The HA redirects the packets towards the FA for the users at the visiting networks. The HA gets the CoA from Binding Table to tunnel the packets appending a new IP header and finally the packets are delivered to the user through the FA by expanding them at the end of the tunnel. Alternatively, on receiving these packets from the user, the FA encapsulates them and tunnels them towards the HA. The HA then expands the packets and sends them to the appropriate destination. This process is known as Triangular Routing that causes some extra delay.

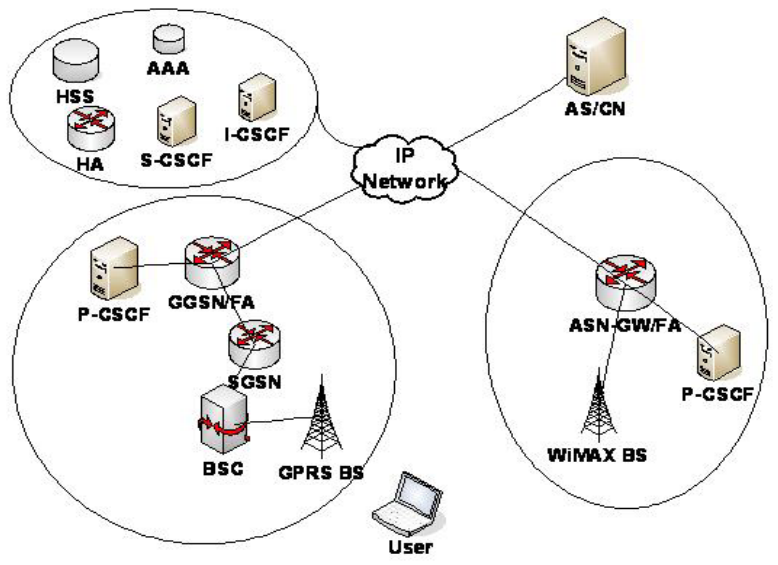

Figure 1. Proposed network diagram.

For the session continuity the application server should be the same for each QoS. The server bandwidth is distributed among the network according to the speed to be supported and server channel frequency is kept less than the channel frequency of the base station so that there remains no in-transit packet in queue during the handover to eliminate the packet loss in the radio path. The base station works as buffer and the length of the buffer depends on the payload of data packets for each of the networks.

\subsection{Working Principle}

The block diagram of our proposed handover algorithm is shown in Fig. 2; assuming the user is currently connected to WiMAX network. GPRS is the other available networks. The user connection to a network is comprises of link layer registration which is followed by the service registration through IMS. After service registration through IMS the user starts getting services. When the user is connected to one network, the mobile device starts monitoring the signal strength of current network and other existing networks receiving Agent Advertisement from respective FAs. The mobile device makes the decision of handover at appropriate moment and then the session for handover to target network starts.

Handoff session in the non-overlapping area is illustrated in Fig. 3. After the completion of link layer registration and service registration by IMS (step 1-3) the user starts getting data through initial network interface. GPRS is considered as the initial interface in this case. When user moves to the edge of a different network, it gets agent advertisement (step 4) sent with the CoA from the foreign agent of the visiting network. It then starts monitoring the signal strength Received Signal Strength Intensity (RSSI) and takes the decision of handover when appropriate. After the decision is taken to handover to a targeted network, the mobile device completes the Link Layer Registration. Link Layer Registration with WiMAX network comprises of Initial Ranging, Session Border Controller (SBC) Negotiation and Registration.

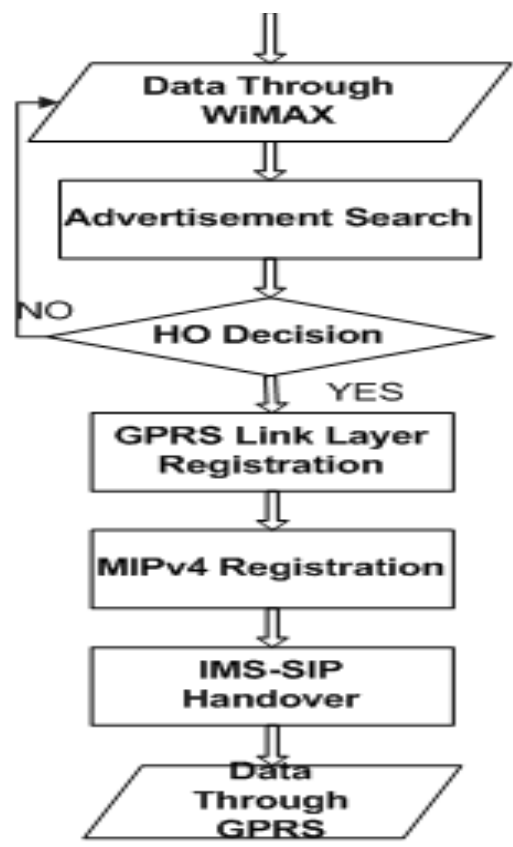

Figure 2. Handover algorithm (WiMAX to GPRS).

At the completion of link layer registration, the user registers the CoA to the home agent (step 5-6) and thus MIP registration is completed. The next stage is the service handover that initiates by the "Re-Invite" message sent from the user to the network with the same user id and session flow information (step 7). When the application server gets this message via the appropriate IMS components, it sends a Session Description Protocol (SDP) message (step 8), "Session Progress", to the user. Upon receiving the SDP, the user responds an acknowledgement message (step 9), "Ack" to the server.

Then the server sends another SDP reply (step 10), "Ok". Now it is time for the user to update the server with another SDP message, "Update" (step 11). When the server successfully updates about the user's current information, it generates "Ok" response to the user (step 12). Finally, the user sends a SDP acknowledgement respond, "Ack" to the server (step 13) and the home agent stops sending data towards initial network and starts sending data through the target network. After the first packet is sent to the FA of target network from the HA, "Binding Update" is done 
which prevents the triangular routing of packets between HA, $\mathrm{FA}$ and $\mathrm{CN}[16]$.

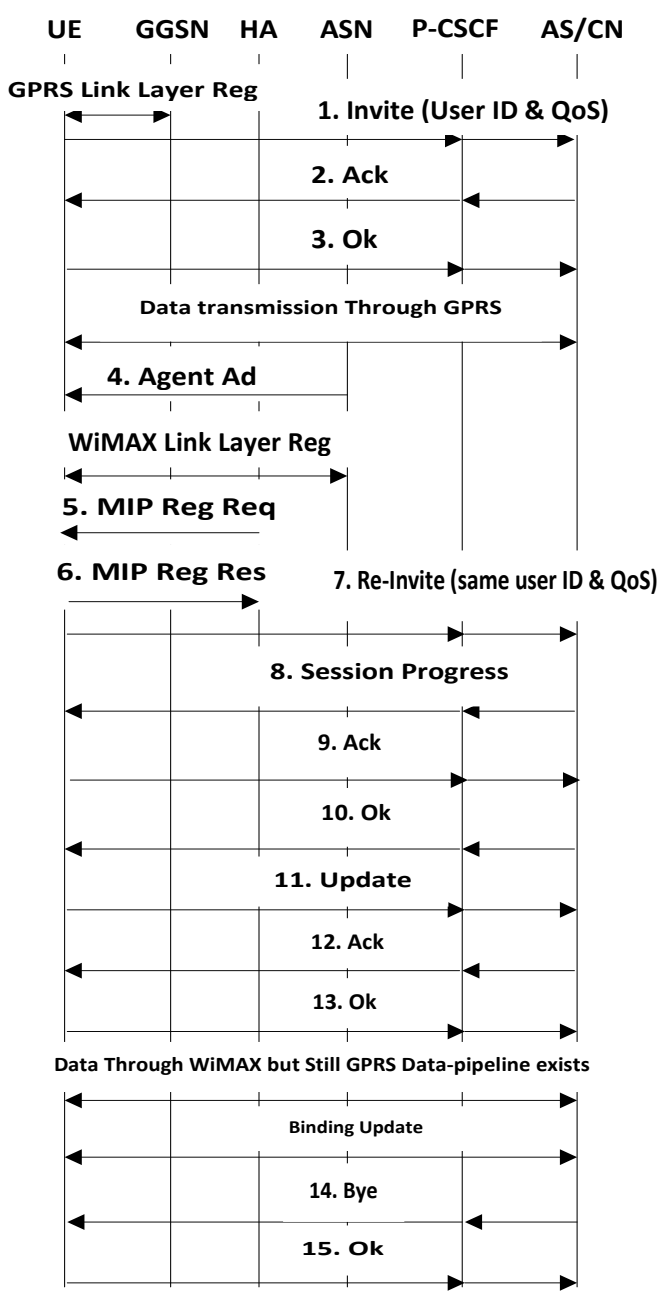

Figure 3. Handoff session in non-overlapping area.

At this stage, the user mobile device is getting data through the target network interface, whilst the data pipeline is not yet broken with the initial network. Henceforth, the user sends a "Bye" request to the initial network to break the data pipeline (step 14-15) and thus the handover is completed.

Thus the user is always connected to some network during handover which will cause no packet loss for handing over. But the user will experience a change in the service data rate when moving from one network access to another.

The handoff session in the overlapping area illustrated at Fig. 4 is different from that of non-overlapping area. When the user moves to an overlapping area, it completes the link layer registration with both the networks with appropriate interfaces and gets the agent advertisement periodically from both the foreign agents (step 1). The user starts getting data through one of the networks that has the stronger signal strength by completing the service registration (step 2-4). The user mobile device is always monitoring the RSSI and takes the decision of handover at an appropriate stage. After that, the mobile device completes the Mobile IP registration (step 5-6) which is followed by the service handover (step 7-13). At this point, data starts moving through the new network. Next step is the "Binding Update" which is followed by the cancellation of data pipeline with the previous network (step 14-15).

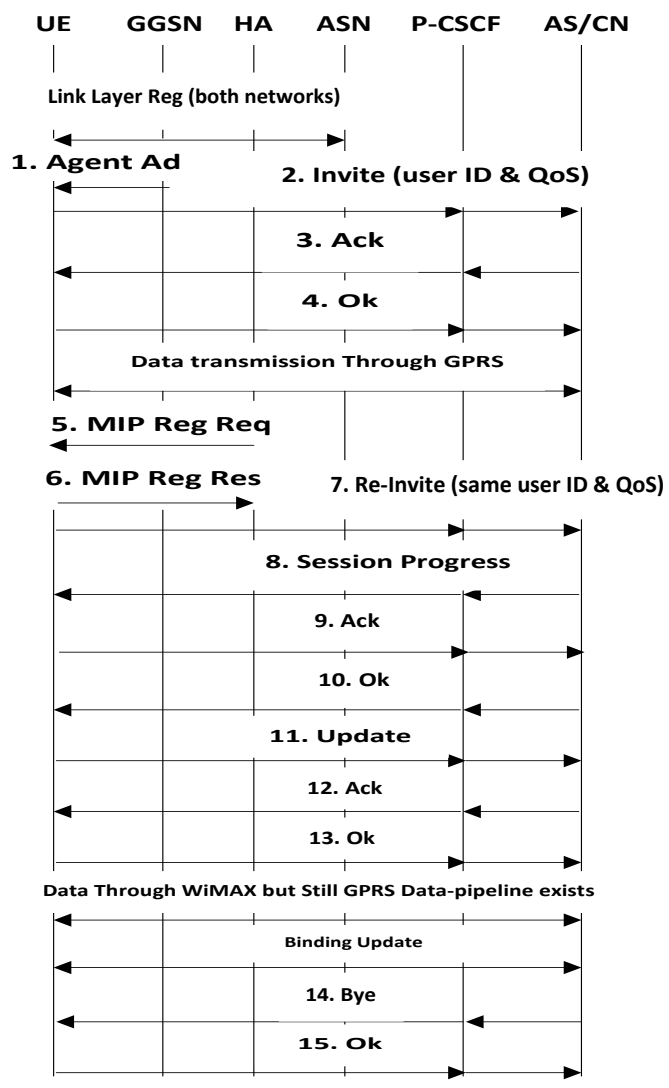

Figure 4. Handoff session in overlapping area.

The latency of handover should be within the specifications defined by IEEE and 3GPP to ensure seamless handover. The latency of the non-overlapping area (the coverage areas of WiMAX and UMTS network do not overlaps each other) consists of the time required for Link Layer Registration, Service Handover and Software Processing Delay. In the overlapping area (the coverage areas of WiMAX and UMTS network overlaps each other), Link Layer Registration is done for all available networks together using their respective interfaces as soon as the user moves into. Thus in overlapping area the delay for handover consists of only Service Handover and Software Processing Delay. The latency for handover in overlapping area and non-overlapping area can be expressed by the equation (1) and (2) respectively.

$$
\begin{aligned}
& \mathrm{T}_{\mathrm{HO}}=\mathrm{T}_{\mathrm{LLR}}+\mathrm{T}_{\mathrm{SH}}+\mathrm{T}_{\mathrm{SPD}} \\
& \mathrm{T}_{\mathrm{HO}}=\mathrm{T}_{\mathrm{SH}}+\mathrm{T}_{\mathrm{SPD}}
\end{aligned}
$$

Where,

$$
\begin{aligned}
\mathrm{T}_{\mathrm{HO}} & =\text { Total Handover Latency } \\
\mathrm{T}_{\mathrm{LLR}} & =\text { Delay for Link Layer Registration } \\
\mathrm{T}_{\mathrm{SH}} & =\text { Delay for Service Handover } \\
\mathrm{T}_{\mathrm{SPD}} & =\text { Software Processing Delay }
\end{aligned}
$$




\section{Performance of the Proposed Architecture}

We have implemented our proposed method described in the previous section by the OPNET Modeler 14.5 and carried out the simulations to characterize the performance of the proposed method of vertical handover.

The standard WiMAX model suite provided by the OPNET Modeler was not competent with our algorithm and the modeller did not provide the GPRS model. Hence, we designed a WiMAX module and GPRS module that supports our algorithm. Finally, we projected a mobile device to support both WiMAX and GPRS interfaces. The scenario developed by OPNET for handover between WiMAX and GPRS is shown in Fig. 5.

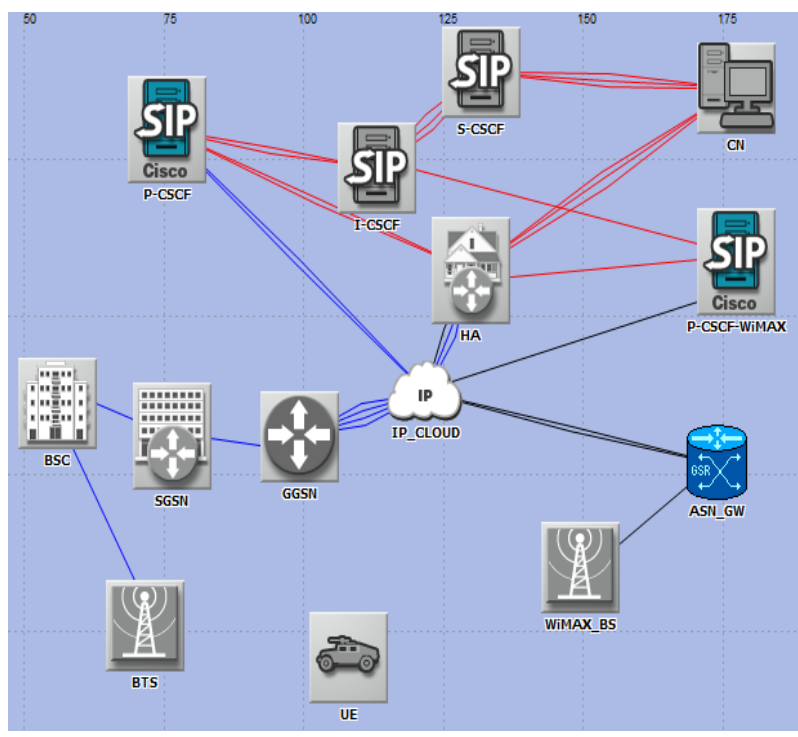

Figure 5. OPNET scenario for handovedr between WiMAX \& GPRS

Table 1. OPNET configuration parameters.

\begin{tabular}{|c|c|}
\hline Parameters & Setting/Description \\
\hline Simulation Period & 400 simulation \\
\hline Agent Advertisement & After half way \\
\hline GPRS data rate & Up to $100 \mathrm{kbps}$ \\
\hline WiMAX data rate & Up to $1.6 \mathrm{mbps}$ \\
\hline Simulation Kernel & Kernel type prefer- \\
\hline Values per statistics & 100 \\
\hline Session starts & After $100 \mathrm{sec}$ \\
\hline Handover starts & After $200 \mathrm{sec}$ \\
\hline QoS type & FTP \\
\hline Service rate & 1 per each node \\
\hline FTP server channel frequency (GPRS) & $10 \mathrm{~Hz}$ \\
\hline FTP server channel frequency (WiMAX) & $1.56 \mathrm{kHz}$ \\
\hline FTP packet size & $1051.5 \mathrm{Byte}$ \\
\hline Packet generation rate & Constant \\
\hline
\end{tabular}

The GPRS network is connected to the IP-Cloud through the GGSN which acts as the FA and the WiMAX network is connected to the IP-Cloud through the ASN-GW, FA for WiMAX Network. Both WiMAX and GPRS networks have their own P-CSCF to get entrance to IP multimedia services. $\mathrm{P}-\mathrm{CSCF}$ connects to the I-CSCF where the registration for the IMS is done and S-CSCF selects the server according to the requested QoS by the user. HA is located in the home network and makes the Mobile IP registration for the user moved to a visiting network.

The simulation was carried out for File Transfer Protocol (FTP) session for handover between WiMAX and GPRS. The latency for the handover in overlapping area and non-overlapping area was measured by the simulation. The parameters configured in the OPNET Modeler are summarized in Table 1.

The average latency for handover from WiMAX to GPRS in overlapping area was $119 \mathrm{~ms}$ and in non-overlapping area was $1.814 \mathrm{sec}$. For handover from GPRS to WiMAX, the latency in overlapping area was $108 \mathrm{~ms}$ and in non overlapping area was $1.920 \mathrm{sec}$. The comparison is shown in Fig. 6 and Fig. 7.

\section{ms Overlapping area}

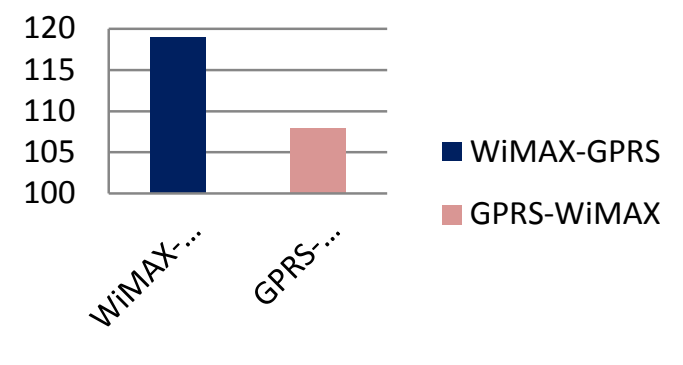

Figure 6. Latency in overlapping area for handover between WiMAX \& GPRS.

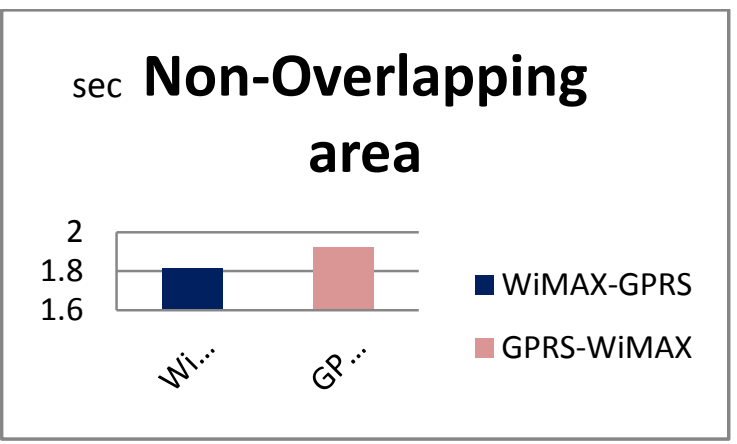

Figure 7. Latency in non-overlapping area for handover between WiMAX \& GPRS.

From the Fig. 6, in overlapping area the handover from WiMAX to GPRS caused higher latency than that from GPRS to WiMAX. This was because the service registration in GPRS is higher than that in WiMAX. But according to Fig. 7, in non-overlapping area the handover from WiMAX to GPRS caused lower latency than that from GPRS to WiMAX. This is because the average delay for GPRS Attach and PDP Activation in GPRS is lower than the average delay for Initial Ranging and WiMAX Registration in WiMAX.

The user experienced an increase in speed when moving from GPRS network area to WiMAX network area as shown 
in Fig. 8. Conversely, the user experienced a decrease in speed when moves from WiMAX area to GPRS area as shown in Fig. 9.

Finally, we measured the end-to-end (ETE) delay in both the networks for FTP and the average ETE delay comparison is shown in Fig. 10.

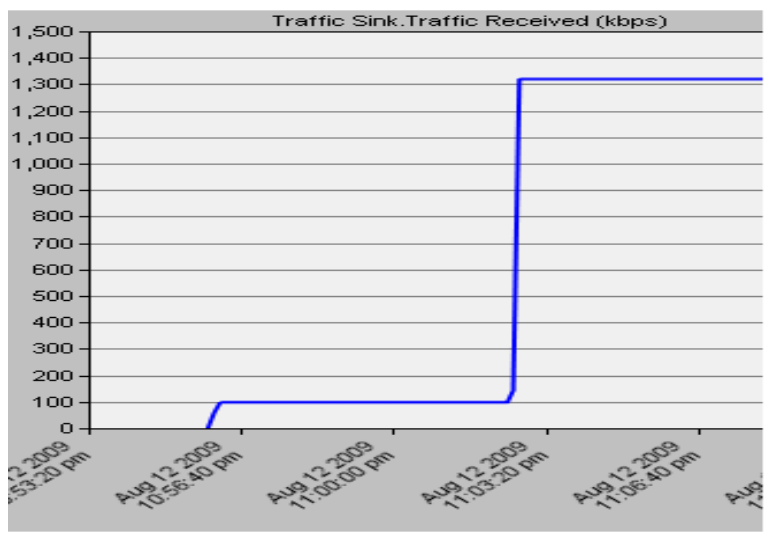

Figure 8. Increase in data rate for handover from GPRS to WiMAX

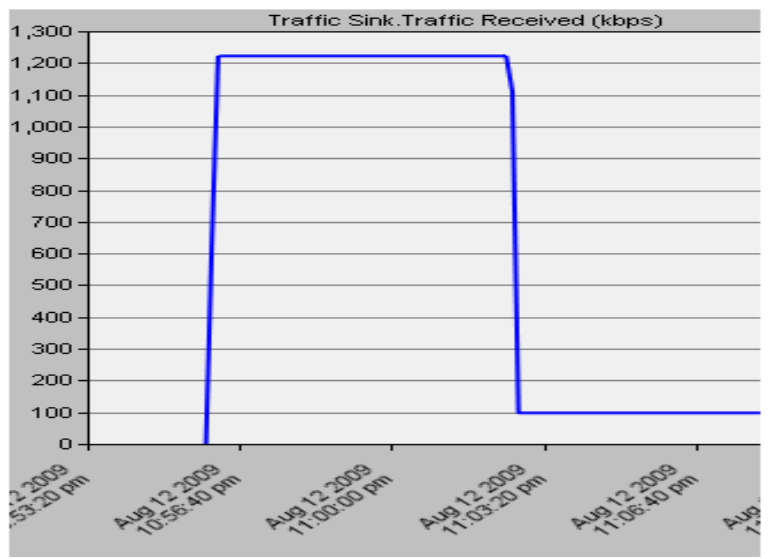

Figure 9. Increase in data rate for handover from WiMAX to GPRS.

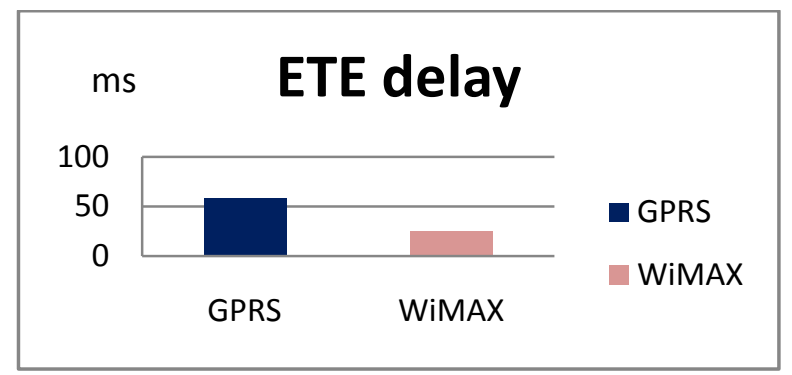

Figure 10. ETE delay.

The average ETE delay was about $57.7 \mathrm{~ms}$ in GPRS and $33.4 \mathrm{~ms}$ in WiMAX. The ETE delay varies according to the pay load of the traffic. Hence, the jitter for FTP session was about $24.3 \mathrm{~ms}$.

\section{Discussion}

The results obtained from our simulation shows that the latency in the overlapping area is very low and good enough to support FTP session without any interruption. On the other hand, the latency in the non-overlapping area is high enough that the on-going session was supposed to be dropped. However, our mobile device with multiple transceivers capable of maintain radio links between WiMAX and GPRS networks simultaneously resulted in a make-before-break type of handover. The service could continue through one interface while the other interface was being used for handing over process. Henceforth, the high latency in non-overlapping area instigated no interruption on the service to the consumers and there was no packet loss during handover in the radio path. At the end of the handover process the server just re-directed the data packets to the new network.

There would be some packet losses due to the flow routing mechanism which was not had to do with the handover mechanism and out of the scope of this article.

\section{Conclusions}

This article narrates a mobility-aware architecture for interworking heterogeneous cellular and wireless networks that enables GPRS and WiMAX systems to manage data user mobility under a common platform with a mobile device able to maintain the dual connection simultaneously. This approach can be a good solution for intersystem handover for the upcoming $4 \mathrm{G}$ networks.

\section{ACKNOWLEDGMENTS}

The authors wish to show gratitude to Suruhanjaya Komunikasi dan Multimedia Malaysia (SKMM) for supporting this research under RCP research grant scheme and to DiGi Telecommunication Sdn Bhd for providing documentations and practical data.

\section{REFERENCES}

[1] IEEE Standard 802.16: Air Interface for Fixed Broadband Wireless Access Systems, 2004

[2] IEEE Standard 802.16e: Air Interface for Fixed and Mobile Broadband Wireless Access Systems, 2005

[3] WiMAX Forum ${ }^{\mathrm{TM}}$, Mobile WiMAX - Part 1: A Technical Overview and Performance Evaluation, August 2006

[4] V. K. Varma, S. Ramesh, K. D. Wong, M. Barton, G. Hayward, and J. A. Friedhoffer, "Mobility management in integrated UMTS/WLAN networks," in Proceedings of the IEEE International Conference on Communications, Anchorage, Alaska, USA, pp. 1048-1053, 11-15 May 2003

[5] Y. Min-hua, L. Yu, and Z. Hui-min, "The Mobile IP handoff between hybrid networks," in proceedings of the $13^{\text {th }}$ IEEE International Symposium on Personal, Indoor and Mobile Radio Communications, Beijing, China, vol. 1, pp. 265-269, 2003 
[6] C. Chang, "A Mobile-IP based Mobility System for Wireless Metropolitan Area Networks," in proceedings of the IEEE International Conference Workshops on Parallel Processing, Oslo, Norway, pp. 429-435, 2005

[7] K. R. Santhi and G. S. Kumaran, "Migration to 4G: Mobile IP based Solutions," in proceedings of the International Conference on Internet and Web Applications and Services, Guadeloupe, French Caribbean, pp. 76-76, 2006

[8] T. Renier, K. L. Larsen, G. Castro and H. Schwefel, "Mis-Session Macro-Mobility in IMS-Based Networks," IEEE Vehicular Technology Magazine, vol. 2, no. 1, pp. 20-27, 2007

[9] C. Gourraud, "Using IMS as a Service Framework," IEEE Vehicular Technology Magazine, vol. 2, no. 1, pp. 4-11, 2007.

[10] J. O'Connell, "Service Delivery within an IMS Environment," IEEE Vehicular Technology Magazine, vol. 2, no. 1, pp. 12-19, 2007

[11] D. Vingarzan and P. Weik, "IMS Signaling over Current Wireless Networks: Experiments Using the Open IMS Core,"
IEEE Vehicular Technology Magazine, vol. 2, issue. 1, pp. 28-34, 2007

[12] K. S. Munasinghe, M. R. Kibria and A. Jamalipour, "Designing VoIP Session Management over Interworked WLAN-3G Cellular Networks," IEEE Wireless Communications, vol. 15, no. 4, pp. 86-94, 2008

[13] K. S. Munasinghe and A. Jamalipour, "Interworking of WLAN-UMTS Networks: An IMS-based Platform for Session Mobility," IEEE Communications, vol. 46, no.9, pp. 184-191, 2008

[14] C. Perkins, "IP Mobility Support for IPv4," IETF RFC 3344, 2002

[15] 3GPP TS 23.228, "IP Multimedia Subsystem (IMS)," Version 6.10.0 Release 6, 2005

[16] E. Schooler, "SIP: Session Initiation Protocol”, IETF RFC 3261,2002

[17] 3GPP TR 23.981, “Technical Specification Group Services and System Aspects; Interworking aspects and migration scenarios for IPv4 based IMS Implementations," Release 6, 2006 